
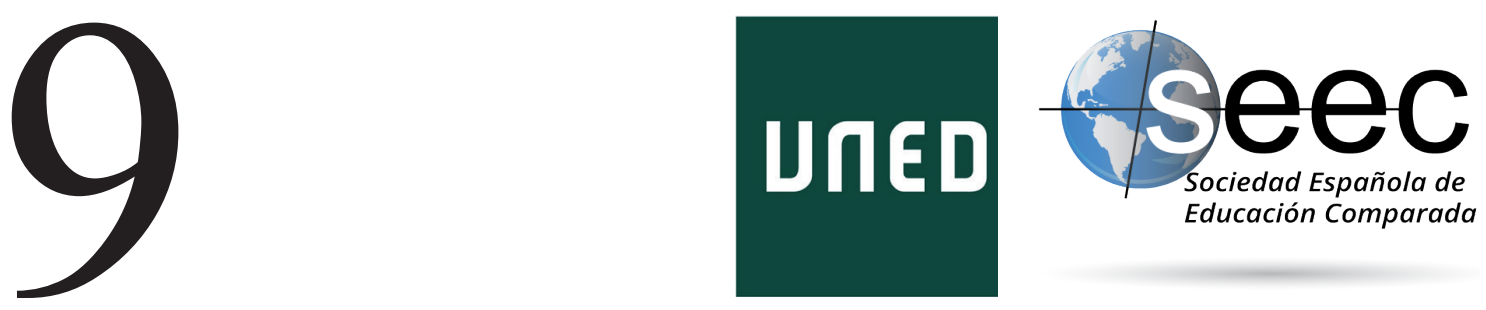

\title{
La cooperación educativa y cultural entre España e India. Logros y retos
}

Educational and Cultural Cooperation between
Spain and India. Achievements and Challenges

Amparo Rodrigo-Mateu*

DOI: $10.5944 /$ reec.39.2021.29093

\section{Recibido: 14 de diciembre de 2010 Aceptado: 11 de mayo de 2021}

\footnotetext{
* Amparo Rodrigo-Mateu: Obtained Masters in Translation and Interpreting, in Audiovisual Communication, in Development Studies and in Teaching Spanish as a Foreign Language. Holds a PhD in Applied Linguistics. Participated in different programs by the Spanish educational action abroad and university cooperation programs in the UK, Austria, Germany, Morocco, USA and India, where she recently focused her research career. Lecturer at the University of Dhaka (Bangladesh), as part of an educational and cultural cooperation project with Universidade de Santiago de Compostela. Member of TALIS Project (Teaching and Acquisition of Solidarity and Intercultural Competences through Languages and Literature), at the University of Valencia.. Datos de contacto: E-mail: mariaamparo.rodrigo@usc.es
} 


\title{
Resumen
}

Este artículo toma como referencia las relaciones educativas y culturales entre España e India a través de dos proyectos oficiales españoles, el programa MAEC*-AECID** de Lectorados de español en Universidades Extranjeras y el Instituto Cervantes. El objetivo es analizar la situación actual y estudiar posibilidades de maximizar la cooperación bilateral en materia educativa y cultural entre España e India, un país que sobrepasa los 1300 millones de habitantes, con una población y una economía en desarrollo, y con un volumen de clase media y una población joven en aumento. Creemos que este impulso por desarrollar un trabajo conjunto y coordinado, el cual debe involucrar también a instituciones indias, permitirá compartir experiencias y conocimientos y favorecerá el compromiso institucional con la educación para el desarrollo sostenible a través de proyectos oficiales de enseñanza de lenguas extranjeras y de difusión cultural en países emergentes.

Palabras clave: cooperación educativa; cooperación cultural; español como lengua extranjera; India

\begin{abstract}
This article takes as a reference educational and cultural relations between Spain and India, based on two official Spanish projects, the MAEC*-AECID** Assistantships Programme to teach Spanish in Foreign Universities and Instituto Cervantes. The objective is to analyse the current situation and study possibilities for maximising bilateral cooperation in education and culture between Spain and India, a country of over 1.3 billion people, with a developing population and economy, and a growing middle-class and young population. We believe that this drive to develop a joint and coordinated action, which should also involve Indian institutions, will enable sharing experiences and knowledge, and will support institutional commitment to education for sustainable development through official foreign language teaching and cultural dissemination projects in emerging countries.
\end{abstract}

Keywords: educational cooperation; cultural cooperation; Spanish as a Foreign Language; India

\footnotetext{
*Ministerio de Asuntos Exteriores, Unión Europea y Cooperación / Spanish Ministry of Foreign Affairs, European Union and Cooperation.

**Agencia Española de Cooperación Internacional para el Desarrollo / Spanish Agency for International Cooperation and Development.
} 


\section{Introducción}

España inició sus relaciones diplomáticas con India en 1956 y, dos años después, abrió su Embajada en Nueva Delhi. Tras sesenta años de relaciones entre ambos países, en 2017, el Real Instituto Elcano de estudios internacionales y estratégicos (España), en colaboración con el instituto de investigación Observer Research Foundation (India), publicó un informe en el que se analiza la situación de las relaciones bilaterales y se señalan los sectores estratégicos que pueden ayudar a fortalecerlas (Campos Palarea y Sengupta, 2017). En dicho estudio se distinguen ámbitos clave de trabajo para unas relaciones que no cuentan en la actualidad con suficientes investigaciones que contribuyan a aprovechar al máximo las oportunidades que existen para la cooperación entre España e India en los ámbitos político, económico, social y, en igual medida, cultural. De esta manera, paralelamente a las dimensiones económica, diplomática, de la seguridad, científica, tecnológica, migratoria y turística, el estudio destaca la importancia que adquieren la educación y la cultura en el marco de las relaciones internacionales entre España y el país asiático, y sugiere una serie de líneas de trabajo entre las que están el fomento de la enseñanza de español como lengua extranjera en India, la difusión cultural entre ambos países, la ampliación de programas institucionales para el intercambio académico y profesional y la formación de profesores indios como docentes de español (Campos Palarea y Sengupta, 2017, pp. 31-33). En conjunto, la educación y la atención a la creciente demanda de conocimientos entre ambos países serían apuestas de futuro que supondrían notables avances en todas las dimensiones abarcadas por la cooperación bilateral y apuntadas más arriba. De acuerdo con lo expuesto, el fortalecimiento de lazos en materia político-económica requerirá, entre otros, de una organización institucional de base que regule la enseñanza de español en India (Rai, 2017, p. 3) y que facilite su desarrollo estructurado.

En la actualidad, la creciente importancia que está adquiriendo la lengua española y su aprendizaje como idioma extranjero (Instituto Cervantes, 2019), queda igualmente manifiesta en India. En el plano de la acción educativa y la cooperación cultural, España realiza su labor institucional en India a través del programa MAEC-AECID de Lectorados en universidades públicas y del Instituto Cervantes. Conscientes de que no responden al mismo tipo de financiación y perfil de alumnado, de mayor poder adquisitivo en el Instituto Cervantes pero no necesariamente en las universidades públicas, este estudio tiene como finalidad suscitar procesos de reflexión que comporten una atención plural al contexto en el que se desarrollan hoy los estudios de español en India, independientemente de los recursos disponibles en cada centro, así como de la procedencia o del perfil socioeconómico del alumnado, y teniendo en cuenta a la sociedad en su conjunto, de acuerdo con el economista indio Amartya Sen (2000, p. 22) al afirmar que el verdadero mérito del desarrollo humano radica en la atención plural.

Atendiendo a estas consideraciones, la intención de este artículo es, en particular, visibilizar la necesidad de cooperación y de coordinación entre las diferentes instituciones involucradas en la enseñanza de español en India. En este sentido, partimos de la idea de una contribución española que, teniendo en cuenta y respetando la realidad y los avances del país en materia educativa, contribuya a establecer y a reforzar las alianzas necesarias de cara a garantizar la calidad y la universalidad de una educación desde la que se fortalezcan valores inclusivos, en un compromiso con la Agenda 2030, y especificamente con el $4 .^{\circ}$ Objetivo de Desarrollo Sostenible (educación de calidad) (Asamblea 
General de las Naciones Unidas, 2015), y que pueda repercutir en nuevas tendencias educativas y en el desarrollo de oportunidades en India, como país emergente en el que todavía persisten marcadas desigualdades sociales. Esta visión de la educación como elemento transformador de la sociedad (Nigam, Ganesh y Rana, 2020, p. 101), a su vez, contribuirá a vertebrar las oportunidades que un país como India - con su vasta extensión, el elevado volumen de población y el rápido crecimiento económico- representa para España (Havlik et al., 2009, p.4). Es aquí donde adquiere importancia la consideración del aprendizaje de español en el ámbito de las relaciones bilaterales entre España e India, donde la creciente demanda de estudios del idioma responde esencialmente a una mayor preocupación por mejorar la calidad de la enseñanza y por ampliar las oportunidades laborales en un mercado cada vez más internacionalizado y en constante expansión (Gómez Sierra, 2019, pp. 5-6), y en el que los programas españoles pueden contribuir positivamente a la configuración de la nueva realidad en enseñanza de lenguas extranjeras, teniendo en cuenta la creciente demanda de estudios de español.

A pesar de esta apuesta por la formación y de la importancia creciente del español y de su estudio en India, encontramos un vacío de información institucional actualizada y unificada que permita generalizar datos acerca del volumen de estudiantes de español a nivel de país, lo cual permitiría establecer comparaciones (UNESCO, 2014, p. 2) y serviría de referencia. Contamos únicamente con los recuentos elaborados y publicados por el Instituto Cervantes, referidos a la demanda existente en su institución. A esta falta de cifras, sumamos la insuficiencia de estudios integrales y exhaustivos sobre el estado de la cooperación cultural entre España e India, así como de investigaciones actualizadas teóricas y prácticas que engloben el conjunto del país y que incorporen un análisis del contexto como factor influyente. Vinculado a esta idea, destacamos la necesidad de considerar aspectos históricos, sociales y cultures, así como aquellos relacionados con la tradición educativa del país, en estudios que, de igual manera, tengan en cuenta la divergencia de visiones de las lenguas que existen entre culturas diferentes y que reflejan diferentes formas de pensamiento (Bernárdez, 2017, p. 9 y p. 12). Estas investigaciones deberían satisfacer, asimismo, la necesidad de colaboración en proyectos internacionales de investigación entre países desarrollados y países en desarrollo. Esto permitiría compartir conocimientos especializados y recursos, intercambiar ideas, establecer y mantener objetivos comunes, desarrollar destrezas, mejorar la productividad y la calidad de los resultados e incrementar las oportunidades de acceso a financiación (Rensburg, Motala y Arulraj, 2015, pp. 815-816).

La presente investigación se sirve de datos oficiales, de estudios e informes previos publicados, de dos cuestionarios distribuidos entre profesorado y alumnado de español en India y, vertebrándolo todo, de información obtenida mediante la observación directa llevada a cabo a lo largo de cuatro años de desempeño profesional en India (tres cursos académicos de lectorado MAEC-AECID en la Universidad de Bombay [2013-2016], y nueve meses de colaboración docente en el Instituto Cervantes de Delhi [2018-2019]). Esta observación participante ha servido para lograr profundizar en la realidad y obtener información cualitativa relevante que ha permitido el análisis interpretativo contrastable de los datos manejados para este estudio (Barro, Jordan y Roberts, 2001, p. 88).

A continuación, se describirá en primer lugar la evolución de India como país emergente, el desarrollo social y de las desigualdades y la influencia que la economía ha tenido en la enseñanza de lenguas extranjeras en el país. Seguidamente, se analizará la situación del español y la evolución de los dos proyectos oficiales de cooperación y de difusión de 
la lengua y la cultura españolas en India -el programa MAEC-AECID de Lectorados y el Instituto Cervantes- y se identificarán aspectos de mejora. Para concluir, a partir del papel que ambos proyectos desempeñan como socios influyentes en el desarrollo educativo en India y en el marco general de las relaciones bilaterales, se formularán propuestas para un trabajo de conjunto que se complemente y se coordine, también en colaboración con instituciones indias, con el fin de alcanzar metas adaptadas a las necesidades desde el desarrollo de programas de enseñanza de idiomas (Gong y Guo, 2019, p. 41) y teniendo en cuenta el contexto actual indio, esto es, una economía emergente con una clase media en crecimiento (Damodar Shingare y Kanoi, 2020, p. 1503) y el aumento significativo de una demanda de estudios de español que apunta hacia la necesidad preeminente de contar con una base institucional coordinada.

\section{India: contexto económico y de enseñanza}

Desde una perspectiva longitudinal, el hispanismo en India cuenta en las últimas décadas con una trayectoria más breve que otros idiomas como el alemán, el francés o el ruso (Ganguly, 2005; Rai, 2017). Hasta 1991, India era una economía mixta controlada por el Estado (Saxena, 2008, p. 117), con tres lenguas extranjeras principales de influencia, esto es, el francés, el alemán y el ruso. Al protagonismo de la lengua francesa en India contribuyó considerablemente el trabajo de difusión lingüística y cultural desempeñado por la Alianza francesa. Por su parte, el auge del alemán se debió al avanzado desarrollo de la industria y economía alemanas y, por último, el ruso adquirió importancia a raíz de las relaciones bilaterales entabladas con Rusia.

La reforma económica y política de liberalización emprendida en 1991 supuso una apertura de la economía india al comercio exterior y a la inversión extranjera, y un aumento de la presencia de empresas extranjeras en el país, con el consiguiente afloramiento de nuevos mercados de trabajo (Saxena, 2008, p. 120) que conllevaron la necesidad de comunicación en lenguas extranjeras, incluyendo el español -junto con el chino, el coreano y el japonés-, debido al desarrollo económico de América Latina y al interés, por parte de India, de fortalecer las relaciones con las economías emergentes del continente (Rai, 2017, p. 2). Estas nuevas exigencias del mercado, inevitablemente, supusieron una serie de reformas en el campo de la educación superior (Saxena, 2008, p. 122) que repercutieron, entre otros, en el auge de la enseñanza de idiomas a nivel universitario, incluido el español. En la actualidad, la demanda de profesionales con conocimientos de idiomas se mantiene en crecimiento especialmente en los ámbitos de la traducción, del turismo y de las tecnologías de la información, de ahí la insistencia en la importancia del aprendizaje de idiomas en todos los niveles de enseñanza reglada (Mathachan, 2019, p. 27-29).

Con 1366 millones de habitantes, según cifras del Banco Mundial para 2019, India es el segundo país más poblado del mundo, por detrás de China. Según los últimos informes del Instituto de Comercio Exterior (ICEX, 2019 y 2020), el volumen de población activa mayor de quince años pasó de un 53,6 \% a un 54,2 \% entre 2019 y 2020, y la tasa de desempleo descendió de un 3,5\% a un 2,6 \% en un país en el que, según el Fondo de Población de las Naciones Unidas, casi la mitad de la población tiene edades inferiores a los 24 años y que además cuenta con el volumen de población joven más grande de su historia (dos tercios del total de la población [Damodar Shingare y Kanoi, 2020, p. 
1503]), una tendencia que las estimaciones prevén que se mantenga hasta $2025^{1}$. De forma similar, según las estadísticas, el volumen de clase media podría superar los mil millones de personas en 2030, un crecimiento del 68,4 \% respecto a cifras de $2019^{2}$. Relacionado de igual manera con el estado de la población, la tasa de población urbana se situaba en el 33,6 \% en 2019 (ICEX, 2019), con un crecimiento anual del 2,3\% según el Banco Mundial ${ }^{3}$, representado mayoritariamente por jóvenes que migran a las ciudades en busca de trabajo (Damodar Shingare y Kanoi, 2020, p. 1505). Para el conjunto de la población del país, se estima un incremento de casi 273 millones de personas entre 2019 y 2050 y se prevé que, alrededor del año 2027, India sobrepase a China en número de habitantes y que se coloque a la cabeza mundial en volumen de población (United Nations Department of Economic and Social Affairs, Population Division, 2019, p. 12). En las mismas circunstancias, el elevado volumen de población joven mencionado, además de ser un motor importante para el futuro económico del país, podría igualmente traducirse en un volumen considerable de mano de obra en la próxima década, situado ya en 520 millones de trabajadores en 2019 y en segunda posición a nivel mundial (Damodar Shingare y Kanoi, 2020, p. 1503).

Desde el punto de vista económico y del desarrollo equitativo de la población, la economía india experimentó una mejora de cuatro décimas en 2007, una evolución que, sin embargo, no se apreció en las cifras de desarrollo equitativo para el conjunto de la población, el cual experimentó un retroceso hasta 2009. Es a partir de entonces cuando observamos un avance gradual positivo en este último indicador, tendencia que no se hace extensiva a los datos relacionados con el declive económico y la pobreza. La puntuación de la economía india alcanzó las cotas más elevadas en 2006, 2009 y 2018 (The Fund for Peace, 2020) ${ }^{4}$ y, según el Programa de las Naciones Unidas para el Desarrollo (PNUD, 2018, p. 32), el coeficiente Gini de desigualdad para India durante el periodo 2010-2017 es de 35,1, una mejora de escasas tres décimas en la distribución de los ingresos familiares con respecto a $2009(35,4)^{5}$.

En lo que respecta a la evolución del grado de alfabetización de la población mayor de quince años, según la Organización de las Naciones Unidas, en 2018, un 74,4 \% de la población estaba alfabetizada, comparado con un 69,3\% en 2011. La evolución por sexos para los mismos años indica un 65,8 \% de hombres alfabetizados en 2011 y un 82,4 \% en 2018. En el caso de las mujeres se ha pasado de un 59,3\% en 2011 a un 78,9\% en $2018^{6}$. Cabe mencionar que el analfabetismo afecta, en mayor medida, a la India rural, medio en el que en 2030 seguirá viviendo la mayor parte de la población (Damodar Shingare y Kanoi, 2020, p. 1505).

Con todo, y a pesar de los contrastes y de las desigualdades que persisten y que afectan al desarrollo equitativo de la población, India cuenta hoy con una de las economías de crecimiento más acelerado del mundo, ha conseguido reducir la pobreza extrema

1 Recuperado el 9 de diciembre de 2020 de https://www.unfpa.org/data/demographic-dividend/IN y https://india.unfpa.org/en/topics/young-people-12

2 Recuperado el 9 de diciembre de 2020 de https://oecd-development-matters.org/2019/05/07/ look-east-instead-of-west-for-the-future-global-middle-class/

3 Recuperado el 9 de diciembre de 2020 de https://data.worldbank.org/country/india?view=chart

4 Recuperado el 18 de noviembre de 2020 de https://fragilestatesindex.org/country-data/

5 Recuperado el 19 de noviembre de 2020 de https://data.worldbank.org/indicator/SI.POV. GINI?locations $=$ IN

6 Recuperado el 11 de diciembre de 2020 de http://uis.unesco.org/en/country/in 
(Kalyani, 2015, p. 44) y es la quinta economía mundial en términos de PIB nominal y la tercera por paridad de poder adquisitivo (Damodar Shingare y Kanoi, 2020, p. 1503), por detrás de China y Estados Unidos (Central Intelligence Agency [CIA], 2019) ${ }^{7}$. Sin embargo, a pesar del robusto crecimiento de su economía, el empleo generado beneficia en mayor medida a la mano de obra que cuenta con estudios y que, por tanto, resulta más productiva, que a los más pobres, un capital humano considerado inferior. Esto genera que, en una de las economías emergentes más importantes del mundo, las condiciones de trabajo para muchos de sus ciudadanos dejen todavía margen para la mejora (Kalyani, 2015, p. 44).

Si consideramos que la educación desempeña un importante papel en el crecimiento de las sociedades, en el caso concreto de la enseñanza superior, esta es un elemento influyente en la reducción de las desigualdades económicas y en el desarrollo de capacidades (Nigam, Ganesh y Rana, 2020, p. 97 y p. 101), frenado este último en India por la falta de calidad en la enseñanza y por la desigualdad en las oportunidades de acceso (Mathachan, 2019, p. 23). De la misma manera que los cambios políticos, económicos y sociales acontecidos en Europa a partir de los años sesenta del siglo xx despertaron nuevas actitudes hacia el aprendizaje de idiomas, tendentes a favorecer el desarrollo personal y la comunicación tanto en el ámbito académico como en el profesional (Aguirre Beltrán, 2016, p. 35), uno de los sectores que mejor refleja en la actualidad el proceso de transformación que está experimentando India es el educativo, influido por el crecimiento económico y comercial del país, por la apertura a mercados extranjeros y por el desarrollo de la clase media y el aumento de las cifras de consumo (Gómez Sierra, 2019, pp. 8-9).

La enseñanza superior en India ha experimentado un crecimiento y una expansión acelerados desde la independencia del país en 1947 (Nigam, Ganesh y Rana, 2020, p. 102), un crecimiento que en el ámbito público, por el contrario, no ha ido acompañado de un desarrollo integral paralelo. 73 años después, la educación terciaria en India sigue haciendo frente a desafíos de desarrollo sostenible como la equidad de género, de clases y de regiones; la calidad de la educación (Damodar Shingare y Kanoi, 2020, p. 1506; Nigam, Ganesh y Rana, 2020, p. 97); las infraestructuras, la inversión en investigación y las oportunidades para la movilidad y el intercambio académico (Sheikh, 2017, pp. 39-40). A todo ello se suma una escasez de personal docente de un 25-30 \%, según datos del Ministerio de Desarrollo de Recursos Humanos (Nigam, Ganesh y Rana, 2020, p. 101). Ante esta coyuntura, India debería plantearse, a partir de un análisis de las necesidades sociales, las capacidades en lenguas extranjeras que quiere desarrollar a nivel de país (Gong y Guo, 2019, p. 22).

La University Grants Commission (en adelante, UGC) nació en 1956 como institución coordinadora de los estudios superiores India. Entre sus funciones están, sumado a la coordinación, la aportación de fondos y el mantenimiento de unos niveles de calidad en las instituciones de educación superior (Nigam, Ganesh y Rana, 2020, p. 98) que permitan afrontar los retos del siglo xxi (Mathachan, 2019, p. 23). Según datos recopilados en su web, el número de centros de enseñanza terciaria en India pasó de 725 en 1951 a más de 43000 en 2017. Un informe elaborado por el Departamento de Educación para el curso 2018-2019 (Ministry of Human Resource Development, 2019, p. i), en el que se recopilan datos aportados por los propios centros, cifra en 51649 el número de centros de enseñanza superior registrados, 1685 más que el año anterior (Ministry of Human

7 Recuperado el 18 de noviembre de 2020 de https://www.cia.gov/library/publications/the-worldfactbook/geos/in.html 
Resource Development, 2018, p. i). 993 de estos centros son universidades, 39931 son colegios universitarios y 10725 son instituciones reconocidas como universidades. De las 993 universidades, 385 son privadas, 394 se ubican en zonas rurales y 9 son institutos universitarios de lenguas. Si comparamos los mismos informes anteriores, el número de matriculaciones en el conjunto de instituciones de enseñanza superior pasó de 36,6 millones en 2017-2018 a 37,4 millones para el curso siguiente. Del total, el 48,6 \% de las matriculaciones durante 2018-2019 corresponden a mujeres, un aumento de un punto respecto a las registradas para el curso anterior. Al mismo tiempo, según estudios de la OCDE (OECD, 2012, p.2, y 2017, p. 10), en 2013 India se situaba por detrás de China y de Estados Unidos en número de graduados universitarios y se estima que en 2030 ocupe el segundo puesto, por detrás de China. No obstante, a pesar de la rápida expansión de las instituciones de enseñanza superior tras la independencia, tres temas que preocupan hoy en el país y que conciernen a la calidad de la educación superior en centros públicos son la insuficiente dotación presupuestaria, la ausencia de políticas sólidas de desarrollo del sector a largo plazo que está beneficiando a las instituciones privadas de enseñanza y el hecho de que ninguna universidad india destaque en las principales clasificaciones mundiales (Nigam, Ganesh y Rana, 2020, p. 101).

Relacionado con la movilidad estudiantil internacional, el Instituto de Estadística de la UNESCO contabilizan más de 375 ooo estudiantes indios en países extranjeros. Los destinos principales son Estados Unidos (135 940 estudiantes), Australia (73 316), Canadá (34 806), Reino Unido (19 599), Alemania (15 473), Emiratos Árabes Unidos (13 370) y Nueva Zelanda $(11604)^{8}$. Un gran reto ahora para las universidades españolas es aumentar la matriculación de estudiantes procedentes de India, situada en la actualidad, según las mismas estadísticas, en 626 estudiantes. Esto indica un número muy reducido de alumnado indio en universidades españolas, a pesar del prestigio que tienen en India instituciones como las escuelas de negocios españolas.

\section{El español en India}

El español es la segunda lengua más hablada del mundo, casi 22 millones de personas lo estudian como lengua extranjera y se disputa con el francés y con el chino mandarín el segundo puesto como segunda lengua extranjera de estudio a nivel mundial (Instituto Cervantes, 2019, p. 12 y p. 19).

Los orígenes de la enseñanza de español en India se remontan a 1958, y tuvieron lugar en instituciones dependientes del Ministerio de Defensa nacional (Ganguly, 2005). En el plano universitario, la Universidad de Delhi cuenta con estudios de español desde comienzos de la década de $1970 \mathrm{y}$, desde los años noventa, con estudios hispánicos a nivel de grado y de máster. Por su parte, la Universidad Jawaharlal Nehru incluyó los estudios hispánicos entre sus programas en el año 1971, con proyecciones docentes e investigadoras. Aunado a esto, cabe agregar las oportunidades para realizar estancias en países hispanohablantes de las que se beneficiaron profesores indios y que contribuyeron a estructurar los estudios hispánicos en India, a los cuales se suma ahora una nueva generación de hispanistas indios jóvenes formados mayoritariamente en India (Pujol Riembau, 2012).

Actualmente existe un interés creciente por el aprendizaje de español a nivel universitario en India, y diversas universidades del país ofrecen estudios de idioma, pero no

8 Recuperado el 24 de noviembre de 2020 de http://uis.unesco.org/en/uis-student-flow 
contamos con un recuento o seguimiento institucional general centralizado que indique cuáles son estas universidades, los niveles de estudios que ofrecen y el volumen de alumnado. Todo ello permitiría obtener una visión de conjunto del país. A estas circunstancias hay que añadir que pocas de estas universidades cuentan con estudios completos de grado y máster. Entre aquellas de las que tenemos constancia están la Universidad Jawaharlal Nehru de Delhi, la Universidad de Delhi, la Universidad de Inglés y Lenguas Extranjeras de Hyderabad, la Universidad Doon de Dehradun y la Universidad Musulmana de Aligarh. La Universidad Savitribai Phule de Pune, por su parte, ofrece únicamente estudios de máster. Existen además otras universidades públicas que imparten cursos de español al margen de los programas de grado ${ }^{9}$, o como asignatura optativa incluida en el plan de estudios de estos, como son la Universidad Hindú de Benarés, la Universidad Jamia Milia Islamia de Delhi, la Universidad de Rajastán, la Universidad de Inglés y Lenguas Extranjeras de Shillong, la Universidad de Hyderabad, la Universidad de Bangalore y la universidad a distancia IGNOU (Indira Gandhi National Open University).

Si a nivel universitario, como detallaremos más adelante, se observa un aumento del volumen de estudiantes para estudios de español, este crecimiento es igualmente destacado en centros privados de enseñanza no reglada de idiomas, entre los que incluimos el Instituto Cervantes (Gómez Sierra, 2019, pp. 13-18). Este último incremento responde a un interés por aprender español, en la mayoría de los casos con miras profesionales, que las universidades no consiguen atender (Pujol Riembau, 2012). Es también en los centros privados de enseñanza reglada primaria y secundaria donde las lenguas extranjeras realmente se valoran y donde, si bien su aprendizaje en India se asocia más al francés y al alemán -ambos con una trayectoria más larga y con mayor presencia en el país-, el español despierta cada vez más interés (Francisco Javier Menéndez Sánchez, comunicación personal, 3 de junio de 2019).

Esta creciente importancia que está adquiriendo el español en la configuración del mapa de las lenguas extranjeras estudiadas en India apunta hacia la necesidad de aumentar la inversión en formación de profesorado de español, más allá del Instituto Cervantes, y en programas de intercambio académico (Campos Palarea y Sengupta, 2017, p. 32), así como de ofrecer oportunidades de movilidad tanto para estudiantes como para docentes.

Llegados a este punto, con el objetivo de determinar la percepción del grado de formación docente y las opciones de movilidad, entre abril y junio de 2019, se distribuyeron sendas encuestas entre alumnado y profesorado de español en diferentes instituciones del país que proporcionaron información cuantitativa relevante para complementar este estudio. De un total de 216 estudiantes participantes procedentes de doce centros de enseñanza, las muestras obtenidas corresponden al Instituto Cervantes de Nueva Delhi, la Universidad de Delhi, la Universidad de Rajastán, la Universidad de Bombay, la Universidad Savitribai Phule de Pune, la Universidad Hindú de Benarés, la Universidad de Inglés y Lenguas Extranjeras de Shillong, la Universidad de Hyderabad, la Universidad Amity de Noida, la Universidad de Bangalore, la Universidad Central de Bangalore y el Central College Bangalore.

En referencia al grupo de docentes, contamos con 38 participantes procedentes de veinte instituciones. Estas son el Instituto Cervantes de Nueva Delhi, la Universidad de Delhi, la Universidad de Bombay, la Universidad Jawaharlal Nehru, la Universidad

9 La docencia de lenguas extranjeras impartida fuera de los estudios de grado se estructura en los tres niveles siguientes: Certificate (niveles A1-A2 del Marco Común Europeo de Referencia para las lenguas), Diploma (niveles A2-B1) y Advanced Diploma o Post-Diploma (niveles B1-B2). 
Hindú de Benarés, la Universidad de Hyderabad, la Universidad de Rajastán, la Universidad Jamia Milia Islamia, la Universidad de Inglés y Lenguas Extranjeras de Hyderabad, la Universidad Savitribai Phule, el Mangement Development Institute de Gurgaon, el Indian Institute of Management de Indore, Indira Gandhi National Open University, la Universidad Amity de Rajastán, la Universidad de Bangalore, el colegio internacional Candor de Bangalore, The International School Bangalore, el colegio internacional Jamnabai Narsee de Bombay y dos institutos de lenguas, el Instituto Hispania y el Lexicos Institute de Gurgaon. De los 38 docentes, 22 (42 \%) son de origen indio y 16 (58\%) de origen hispano.

Por lo que se refiere a la movilidad, el 91 \% del alumnado encuestado, esto es, 197 participantes, afirma no haber estado nunca en un país de habla hispana. De los 19 restantes, en 11 casos el motivo de la visita ha sido académico. Del total de los encuestados, tan solo un 3,2 \% (7 participantes) ha estudiado algún curso de español en un país de habla hispana y, entre aquellos que cursan estudios formales, un $59 \%$ (127 estudiantes) mantiene que sus centros de estudio no les proporcionan oportunidades para participar en estancias de formación en países hispanohablantes.

Del lado de los profesores, 13 (un $59 \%$ ) de un total de 22 docentes indios encuestados afirman haber participado en algún programa de formación en un país hispano. Con la excepción de cinco que trabajan en instituciones fuera de la capital, los ocho restantes ejercen sus funciones en Delhi. De estos ocho, seis lo hacen en el Instituto Cervantes.

\subsection{Programas españoles de enseñanza de lengua y difusión cultural en India}

La labor de acción exterior que España desarrolla en India en materia educativa y cultural se desempeña principalmente a través de dos programas oficiales: el programa MAEC-AECID de Lectorados en universidades indias y el Instituto Cervantes, presentes en el país desde $2006^{10}$ y 2009, respectivamente, y adscritos al Ministerio de Asuntos Exteriores, Unión Europea y Cooperación.

\subsubsection{Programa MAEC-AECID de Lectorados}

El Programa MAEC-AECID de Lectorados de español en universidades extranjeras se ofrece, en convocatorias anuales, a ciudadanos españoles. En el caso de India, son cuatro las instituciones que coordinan su implementación. Se trata de dos instituciones españolas (la AECID y la Sección Cultural de la Embajada española en Nueva Delhi) y dos organismos indios (la UGC y las mismas universidades que participan en el programa). Su implementación queda regulada por un acuerdo multilateral renovable firmado entre la Embajada de España y las distintas universidades.

Para este último año académico, 2019-2020, se han convocado siete plazas de lectorados de español en las siguientes universidades indias: la Universidad de Rajastán, la Universidad Savitribai Phule de Pune, la Universidad de Benarés, la Universidad de Inglés y Lenguas Extranjeras de Hyderabad y en tres universidades de Delhi, la Universidad Jawaharlal Nehru, la Universidad de Delhi y la Universidad Jamia Milia Islamia $^{11}$. Todas ellas se corresponden con las mismas plazas convocadas para el curso

10 La primera convocatoria oficial a la que hemos tenido acceso corresponde al curso académico 2006-2007. Según información proporcionada por la AECID, la Universidad de Delhi y la Universidad Jawaharlal Nehru, también en la capital, participan en el programa con anterioridad a 2003.

11 Recuperado el 12 de diciembre de 2020 de https://www.aecid.es/ES/becas-y-lectorados/ convocatorias-maec-aecid/lectorados 
anterior, 2018-2019. Únicamente la Universidad Jawaharlal Nehru, la Universidad de Delhi y la Universidad de Inglés y Lenguas Extranjeras de Hyderabad incluyen entre sus programas estudios hispánicos a nivel de grado, de máster y también de tercer ciclo.

En términos generales, el número de matriculaciones confirma una gran demanda de aprendizaje de español y de especialización a nivel universitario, cifras que son especialmente significativas en los cursos correspondientes a los niveles más bajos del idioma, según hemos constatado a partir de los datos de matriculación proporcionados por diferentes departamentos universitarios. Así, el Departamento de Francés de la Universidad de Bombay registró una media de 70 estudiantes para el nivel Certificate de español entre 2013 y 2016, último año en el que participó en la convocatoria de lectorados, y la Universidad de Benarés hubo una media de 60 entre 2017 y 2019. Por su parte, el Departamento de Lenguas Germánicas y Romances de la Universidad de Delhi contó en 2019 con 119 alumnos matriculados en estudios de grado y 32 de máster, ambas cifras que han ido aumentando progresivamente en los últimos años. En el Centro de Estudios Españoles, Portugueses, Italianos y Latinoamericanos de la Universidad Jawaharlal Nehru, desde 2017 hasta 2019, el número de estudiantes de grado ha pasado de 30 a 39. Por último, en la Universidad de Rajastán, entre 2017 y 2020, el número de estudiantes para los cursos de Certificate y de Diploma ha aumentado de 46 a 82 y de 11 a 25, respectivamente.

A pesar de este manifiesto interés por los estudios de español, las diferentes convocatorias anuales de lectorados publicadas por la AECID demuestran un descenso paulatino del número de puestos convocados. De las diez plazas convocadas para el curso académico 2006-2007 se pasó a doce en el curso siguiente, 2007-2008. Sin embargo, a partir de aquí empiezan a disminuir gradualmente hasta situarse en las siete plazas ofrecidas en la última convocatoria. Cabe destacar que, en un periodo de tiempo de dos años (desde 2017 hasta 2019) se cancelaron las plazas de lectorado en tres de las cuatro ciudades con mayor volumen población del país, esto es, Bombay, Calcuta y Chennai. Este retroceso ha supuesto un freno para la consolidación del programa de lectorados en India, un programa que, además de beneficiar a las universidades públicas porque les permite contar con profesorado nativo del idioma, abre el camino a la inserción del hispanismo en universidades que no cuentan con una larga trayectoria en estudios de español (Pujol Riembau, 2012). Esta disminución progresiva de las plazas convocadas en los últimos años ocurre precisamente con posterioridad a la difusión de los resultados de la investigación llevada a cabo por el Real Instituto Elcano de estudios internacionales y estratégicos (Campos Palarea y Sengupta, 2017), en la que, en el marco de la mejora de las relaciones bilaterales en materia educativa entre España e India, se cita al Instituto Cervantes pero no se hace mención alguna al programa MAEC-AECID de Lectorados, a pesar de estar ambos adscritos al mismo ministerio y de contar este último con más años de trayectoria docente en el país.

\subsubsection{Instituto Cervantes}

El Instituto Cervantes de Nueva Delhi empezó sus funciones en India en 2009, año en el que se contabilizaron más de 50.000 horas/alumno (Pujol Riembau, 2012). Once años después, en 2018, la cifra había aumentado más de un $700 \%$, hasta alcanzar las 382.000 horas/alumno. Sumado a los Institutos Cervantes de Pekín y de Manila, el centro de Delhi lidera el número de horas de docencia impartidas (Instituto Cervantes, 2018, p. 20 y p. 253). 
Los exámenes oficiales DELE (Diploma de Español como Lengua Extranjera), organizados desde el Instituto Cervantes de Nueva Delhi, se realizan en India desde 2005, gestionados en aquel momento desde el exterior. De los primero 42 candidatos presentados, el número ha ido ascendiendo hasta sobrepasar los 1600 en 2019. Este incremento es una evidencia clara y documentada del progresivo aumento en la demanda de conocimientos de español en India. A continuación, la figura 1 muestra el progreso del volumen de examinandos:

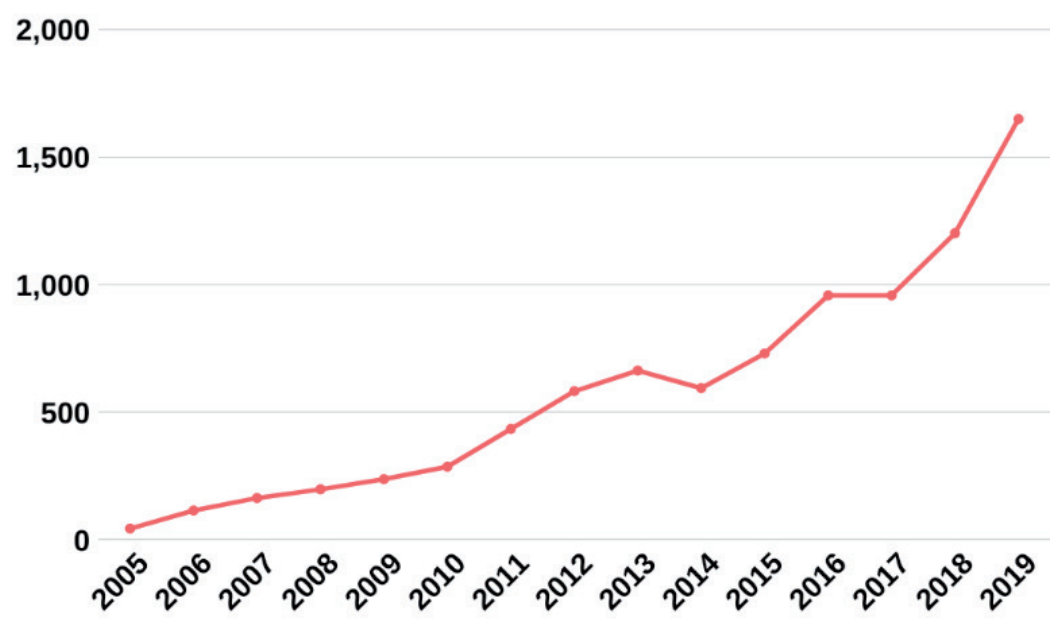

Figura 1. Evolución del número de matrículas para exámenes DELE en India. Fuente: Instituto Cervantes.

En la actualidad, el Instituto Cervantes de Nueva Delhi administra las pruebas de exámenes DELE que se realizan en el mismo centro, en otros centros acreditados en las ciudades indias de Calcuta, Bombay, Jaipur, Musoorie y Chennai, así como en las ciudades de Daca, en Bangladés, y en Lahore, Pakistán. Conviene subrayar que, en el impulso de estas pruebas oficiales de certificación en India, el programa MAEC-AECID de Lectorados desempeña un papel importante (Pujol Riembau, 2012).

El Plan curricular del Instituto Cervantes (PCIC) (Instituto Cervantes, 2006) es el instrumento de referencia en el que se recopilan los objetivos institucionales y docentes de la institución. Entendido como marco de decisión y de actuación, el PCIC representa, desde un enfoque curricular, una visión amplia de los procesos de enseñanza y aprendizaje de idiomas (Aguirre Beltrán, 2016, p. 37). Nace ante la proliferación de centros Cervantes en diferentes países y tiene como meta establecer una serie de criterios generales que permitan implementar una actuación homogénea y coordinada para el estudio del español como lengua extranjera, además de contribuir a una formación docente de calidad (PCIC, 2006, p. 7 y p. 9), adaptando todo ello a cada contexto sociocultural y de enseñanza específicos (Aguirre Beltrán, 2016, p. 54). Desde una perspectiva académicocientífica, el PCIC destaca la necesidad de que el Instituto Cervantes participe en investigaciones internacionales en el campo de las lenguas y en proyectos para el desarrollo de herramientas que permitan intercambiar conocimientos (PCIC, 2006, p. 14 y p. 16).

En cuanto al número de centros docentes, si tomamos como referencia la presencia de institutos análogos en India, el Instituto Cervantes dispone únicamente de un centro, 
comparado con los catorce institutos de Alliance française, los seis del Goethe-Institut y los dos centros del Instituto Camões. Teniendo en cuenta que la presencia de centros Cervantes en India es inferior a la de sus análogos europeos y que la oferta de estudios de español en India es inferior a la demanda actual, se vuelve necesaria la expansión del trabajo que realiza el Instituto Cervantes a otras ciudades del país como Calcuta o Bombay (Campos Palarea y Sengupta, 2017, pp. 31-32), las capitales cultural y financiera, respectivamente. Al mismo tiempo, es necesario extender el núcleo de la enseñanza de español, concentrado en la actualidad en el Instituto Cervantes y, por tanto, en Nueva Delhi. No obstante, como hemos comprobado, el segundo proyecto institucional para la enseñanza de español en India, el programa MAEC-AECID de Lectorados, ha perdido fuerza en los últimos años.

\subsection{Limitaciones del estudio}

Describimos, a continuación, las tres limitaciones metodológicas principales surgidas durante la realización de este estudio, en relación con los dos cuestionarios distribuidos.

En primer lugar, con el objetivo de ampliar la muestra de estudio más allá de las instituciones en las que se ha realizado o se estaba realizando un trabajo presencial, y ante la falta de información sistematizada sobre las universidades indias que ofrecen estudios de español, hemos recurrido a contactos directos establecidos durante los años de experiencia personal docente en el país para que nos facilitaran el acceso al profesorado y al alumnado de sus respectivos centros, así como a una búsqueda general en páginas web de universidades y departamentos universitarios. En el caso del cuestionario para el profesorado, además, se ha ampliado la búsqueda a través de redes sociales profesionales y de la base de datos de la Asesoría de Educación de España en India, la cual cuenta con las direcciones de contacto de los docentes de español en India registrados. Esta última, sin embargo, es general y no permite filtrar la búsqueda por tipo de centro de trabajo. Se ha tenido que especificar en la toma de contacto, por tanto, que el cuestionario iba dirigido a profesorado universitario. A pesar de esto, han participado cinco docentes que desempeñan su labor en institutos de idiomas o colegios internacionales. Sus respuestas han sido finalmente incluidas en las muestras de estudio atendiendo al razonamiento siguiente:

Primero, consideramos que aportan información relevante desde una perspectiva complementaria. Segundo, a lo largo de su formación han estado o están en contacto con instituciones de enseñanza superior o de enseñanza a adultos de español. Por último, conforman una red de trabajo alrededor de la enseñanza de español en India, a través de su suscripción a la lista de distribución de información gestionada por la Asesoría de Educación.

La segunda limitación está relacionada con la comunicación en línea. El hecho de no disponer de acceso directo a la mayoría de los encuestados ha influido en gran medida en la gestión, en la efectividad de los recordatorios posteriores enviados y en el volumen de respuestas recibidas. Este factor ha tenido mayor incidencia en los cuestionarios distribuidos entre alumnado a través de un docente del mismo centro, en calidad de intermediario.

En tercer lugar, recurrir a la distribución de los cuestionarios en línea ha supuesto, indiscutiblemente, una menor inversión de tiempo de cara a la compilación y sistematización de los datos obtenidos, un aspecto positivo teniendo en cuenta la vasta extensión geográfica de India. No obstante, esta modalidad produce, por regla general, un menor índice de respuesta que en situaciones de administración presencial de cuestionarios y también posibles incoherencias en respuestas que, al no tener contacto directo con los participantes, no es posible resolver y podría influir en el grado de fiabilidad y validez de la herramienta. 


\section{Conclusiones}

$\mathrm{Al}$ igual que ocurre en muchos otros países, la lengua española está adquiriendo cada vez más importancia en India, no solo en el mundo académico, sino también en los negocios, y esto genera un aumento de la demanda por su aprendizaje. Si la tendencia de la demanda de aprendizaje de un idioma es un reflejo de la importancia de este en el futuro, en el caso concreto de India, necesitamos contar con información objetiva, sistematizada y actualizada que ayude a diseñar un sistema de centros, a contabilizar el número de estudiantes y a analizar la evolución de los estudios de español en el conjunto del país y en los diferentes niveles educativos. Esto permitirá priorizar objetivos en aras del fortalecimiento de la cooperación educativa y cultural, como pilar influyente en las relaciones bilaterales entre España e India, con miras a largo plazo y teniendo en cuenta las optimistas previsiones de futuro para el español como lengua extranjera de estudio y de comunicación internacional.

La demanda actual de estudios de español en India brinda oportunidades y abre nuevas vías de trabajo para los programas españoles de lengua y cultura ya emprendidos en el país. El aprovechamiento adecuado de esta situación favorable requiere maximizar la cooperación entre las diferentes instituciones españolas involucradas y también entre estas y las instituciones indias. Esto permitirá compartir y comparar datos y experiencias de cara al desarrollo cooperativo de los proyectos de enseñanza y a la capacitación de las instituciones para actuar como referentes de nuevas tendencias educativas, dentro y fuera del país, en calidad de socios dentro de un programa de desarrollo educativo conjunto que permita coordinar enfoques para la evolución del hispanismo que abarquen al conjunto del país, considerando el contexto y las posibilidades de desarrollar programas adaptados a la realidad del momento.

A nivel nacional, sin embargo, encontramos una base institucional insuficiente, no coordinada y centrada principalmente en la capital, Nueva Delhi. Desde el lado institucional español, sería conveniente recobrar e incrementar el número de plazas de lectorados en universidades públicas. Cabe destacar que, de los siete actuales, tres se encuentran en la capital y que se han cancelado tres plazas en universidades de ciudades periféricas importantes como Calcuta, Chennai y Bombay, esta última con representación institucional española a través del Consulado General, de la Consejería de Economía y Comercio y de la Consejería de Turismo. Por su parte, el desarrollo de un programa de trabajo colaborativo entre el Instituto Cervantes y el programa MAEC-AECID de Lectorados permitirá dotar a todas las universidades participantes en este último, sin limitarlas a las de la capital, de un marco institucional más amplio que beneficiaría a la proyección de la lengua española y su(s) cultura(s) en India, y a una consolidación de la labor de los lectores que integre en igual medida el trabajo realizado fuera de la capital.

Asimismo, consideramos que el Instituto Cervantes, además de analizar la idoneidad de contar con un centro para la difusión de la lengua y la cultura españolas en Bombay, capital financiera del país y con un volumen elevado de población joven activa, podría desempeñar un papel determinante a la hora de establecer alianzas con universidades y otras instituciones de educación superior con fines docentes, contribuir con investigaciones al hispanismo académico y cooperar con instituciones indias para establecer líneas e intereses prioritarios de investigación. Todo ello permitirá explotar sinergias y contribuirá a un desempeño más coordinado y a un mejor aprovechamiento de la inversión oficial española en proyectos educativos y culturales en India que, a su vez, comporte beneficios a largo plazo para el conjunto de la cooperación bilateral. 
Desde las instituciones indias, en el contexto del auge del español en el continente asiático, conviene considerar la relevancia que está adquiriendo el estudio del idioma en India y esforzarse por crear un sistema de trabajo coordinado y una oferta universitaria que apueste por un mayor impulso de los estudios de español, por contar con profesorado nativo en la medida de las posibilidades, por el intercambio académico y profesional y por el desarrollo de la investigación, al igual que está haciendo China desde hace ya un tiempo (Marco Martínez y Lee Marco, 2010). Paralelamente, las instituciones de enseñanza superior indias deberían tener las miras puestas también en el desarrollo que está experimentando el español a nivel de Asia y unirse a él. Dentro del continente asiático, India debería hacer muestra de un hispanismo de calidad, derivado de un esfuerzo conjunto y, considerando las tasas de demanda, liderar proyectos académicos y de investigación en el continente, con miras a largo plazo.

Si partimos de la consideración de India como economía emergente, con una población que supera los 1300 millones de habitantes, en pleno desarrollo, con una clase media en aumento formada mayoritariamente por jóvenes y con un descenso gradual del desarrollo desigual de la población desde 2010, se vuelve necesaria una implicación institucional multilateral para analizar pormenorizadamente la situación actual en la que se encuentra la enseñanza de español y el interés por ella, y compartir esta información para que India destaque en informes comparativos internacionales, así como llevar a cabo estudios comparativos sobre la situación de la enseñanza de diferentes idiomas y, en definitiva, potenciar investigaciones que, a partir de una base teórica, analicen los cimientos sobre los que se asientan los estudios de español y que, a su vez, reflejen los distintos contextos y situaciones de enseñanza que se dan a lo largo de toda la geografía del país.

Ante todo, cualquier enfoque para la difusión y organización de los estudios de español en India debe partir de un análisis integral del estado de la cuestión a nivel nacional y, a partir de aquí, establecer un estándar para la enseñanza y aprendizaje del idioma. Esto abrirá nuevas vías de trabajo adaptadas a la situación actual y a las necesidades del país, así como necesarias vías de investigación que permitan la transferencia de resultados replicables y una evolución de los estudios empíricos en el campo de la enseñanza de español en India.

Relacionado con la mejora de las oportunidades de movilidad estudiantil entre India y España, y atendiendo tanto al incremento de estudios de español en enseñanza superior como al volumen mundial que representa el sistema universitario indio, sería conveniente analizar el perfil de alumno que estudia español en las universidades y analizar si procede de estudios hispánicos o de otras titulaciones. Habría que determinar también las características de los alumnos universitarios indios que, bien estudian en universidades españolas, bien muestran interés en ello, y conceder más oportunidades para intercambios académicos. Al mismo tiempo, sería de gran utilidad y conveniencia confeccionar guías con información útil y disponible para aquellos indios atraídos por cursar estudios en universidades españolas que, paralelamente, ofrezca una visión atractiva del sistema educativo español en India. Cabe recordar que Alemania es, en la actualidad, el cuarto país de destino elegido por los estudiantes indios y, a diferencia del inglés, el alemán en India es una lengua extranjera de estudio. Esto demuestra que el alumnado indio interesado en realizar estudios en otros países está dispuesto a aprender idiomas. Cualquier inversión en enseñanza de español en India será, por tanto, una aportación de valor de cara al acercamiento a India del sistema de enseñanza español. La contribución a este tipo de estudios y la mejora de las relaciones con instituciones extranjeras por 
parte de las instituciones indias contribuirían también a aumentar el número de programas de intercambio.

En el caso del profesorado, la inversión en programas de formación en India homogeneizados y coordinados, así como en su movilidad y formación internacional, permitirá continuar vertebrando el hispanismo indio, teniendo en cuenta el contexto actual de internacionalización y de desarrollo del país y de sus programas de enseñanza.

Antes de concluir, merece recordar que, a pesar de las cifras de crecimiento positivas, India representa también una suma de desarrollos a diferentes velocidades. Cobra especial relevancia, por tanto, la necesidad de apostar por el sector público educativo como derecho ciudadano y priorizar proyectos que ayuden a alcanzar la igualdad de acceso a una educación de calidad e inclusiva y que tengan un impacto positivo en el progreso de la educación, en la igualdad de oportunidades, en el desarrollo conjunto de la población y en la lucha contra cualquier desigualdad. Son estas desigualdades las que justifican el programa MAEC-AECID de Lectorados en universidades públicas y su contribución al desarrollo de capacidades en los estudiantes. Creemos que su consideración e inclusión en estudios estratégicos de cooperación bilateral entre España e India les atribuiría la importancia que tienen y, además, evidenciaría el conjunto del trabajo que realiza el Ministerio de Asuntos Exteriores, Unión Europea y Cooperación en India a través de su política de cooperación y difusión educativas y culturales en el exterior.

Por parte de las instituciones españolas que contribuyen a la labor de difusión de la enseñanza de español en India, es necesaria su unidad para explorar el potencial de la lengua española en el país y analizar tanto el estado en curso como el futuro que puede tener como lengua extranjera. Conviene partir de los logros ya alcanzados y avanzar adaptándose a las características educativas, sociales y tecnológicas que presenten los diferentes contextos de enseñanza, olvidando la fórmula de la solución única y valorando el potencial de incluir a India en los discursos internacionales sobre el futuro de la enseñanza de español. Esto, unido al tratamiento de las lenguas como práctica cultural y social, favorecerá procesos de internacionalización de la educación equitativos y equilibrados también fuera de las potencias económicas occidentales y, en la misma línea, contribuirá al cumplimiento de los Objetivos de Desarrollo del Milenio mediante una mejora de la educación de calidad y el acceso universal a ella, en un compromiso por la excelencia.

Para concluir, el español en India queda respaldado por la intensa labor de proyección del idioma en el exterior llevada a cabo desde las instituciones españolas y, además, cuenta con las condiciones económicas propicias por las que atraviesa el país. Todo ello compone un escenario favorable de futuro para la enseñanza del español en India (Ganguly, 2005), un futuro que, en las circunstancias actuales, requiere de actores y de acciones coordinados, que se complementen entre ellos y que trabajen por una expansión unificada del idioma como lengua extranjera de aprendizaje y de comunicación.

\section{Referencias}

Aguirre Beltrán, B. (2016). Análisis de necesidades y diseño curricular. En J. Sánchez Lobato e I. Santos Gargallo (Dirs.), Vademécum para la formación de profesores: Enseñar español como segunda lengua (L2)/lengua extranjera (LE) (vol. II, pp. 35-56). Madrid: SGEL. 
Asamblea General de las Naciones Unidas (2015). Transformar nuestro mundo: la Agenda 2030 para el Desarrollo Sostenible, 21 de octubre de 2015, A/RES/70/1. Recuperado el 21 de noviembre de 2011 de https://www.un.org/ga/search/view_ doc.asp?symbol=A/RES/70/1\&Lang=S

Bernárdez, E. (2017). Non-Western Concepts of Language: Are They of Any Use for the Scientific Study of Language? Complutense Journal of English Studies, 25 (pp. 9-25).

Barro, A., Jordan, S. y Roberts, C. (2001). La práctica cultural en la vida cotidiana: el estudiante de idiomas como etnógrafo. En M. Byram y M. Fleming (Eds.), Perspectivas interculturales en el aprendizaje de idiomas (pp. 82-103). Cambridge: Cambridge University Press.

Campos Palarea, R. y Sengupta, J. (2017). España y la India: en busca de unas relaciones bilaterales más estrechas. Madrid: Real Instituto Elcano.

Damodar Shingare, A. y Kanoi, T. (2020). India 2030: The Decade Ahead. International Journal for Research in Applied Science \& Engineering Technology (IJRASET), 8 (VII), 1503-1514. DOI: https://doi.org/10.22214/ijraset.2020.30458

Ganguly, S. P. (2005). Los estudios hispánicos en la India. En Anuario del Instituto Cervantes 2005 (pp. 429-438). Madrid: Instituto Cervantes.

Gómez Sierra, F. (2019). El mercado de la enseñanza de español en India. Bombay: ICEX.

Gong, T. y Guo, Y. (2019). A Critical Review of China's Foreign Language Program Development: The Perspective of Language Needs. Círculo de Lingüística Aplicada a la Comunicación 79, 21-44.

Havlik, P., R. Stollinger, O. Pindyuk, G. Hunya, B. Dachs, C. Lennon, M. P. Ribeiro, J. Ghosh, W. Urban, V. Astrov, and E. Christie (2009). EU and BRICs: Challenges and opportunities for European competitiveness and cooperation. Brussels: European Commission.

Instituto Cervantes (2006). Plan curricular del Instituto Cervantes. Niveles de referencia para el español. Madrid: Biblioteca Nueva.

Instituto Cervantes (2018). Memoria 2017-2018. Madrid: Instituto Cervantes.

Instituto Cervantes (2019). El español: una lengua viva. Madrid: Instituto Cervantes.

InstitutodeComercio Exterior(ICEX)(2019). Fichas-País 2019. India. Recuperadodehttps:// www.icex.es/icex/es/Navegacion-zona-contacto/libreria-icex/PUB2019817821. html ?idTema $=10707000 \&$ idColeccion $=12060359$

Instituto de Comercio Exterior (ICEX) (2020). Fichas-País 2020. India. Recuperado de https://www.icex.es/icex/es/navegacion-principal/todos-nuestros-servicios/ informacion-de-mercados/paises/navegacion-principal/el-mercado/estudiosinformes/DOC2016645918.html?idPais=IN\&null 
Kalyani, M. (2015). Labour Market Situation in India: An Analysis. International Journal of New Technology and Research (IJNTR), 1(8), 44-52.

Marco Martínez, C. y Lee Marco, J. (2010). La enseñanza del español en China: evolución histórica, situación actual y perspectivas. Revista Cálamo FASPE (56), 3-14.

Mattachan, K. J. (2019). Draft National Educational Policy 2019 and Foreign Language Education in India: A Perspective. Indian Scholar (6), I, 23-30.

Ministry of Human Resource Development, Deparment of Higher Education (2018). All India Survey on Higher Education 2018-2019. Delhi: Ministry of Human Resource Development.

Ministry of Human Resource Development, Deparment of Higher Education (2019). All India Survey on Higher Education 2018-2019. Delhi: Ministry of Human Resource Development.

Nigam, D., Ganesh, M. P. y Rana, S. (2020). Review of the Expansion of Higher Education in India: Cardinal Concerns in the Traverse. Journal of Critical Reviews, 7, 97-102.

Organisation for Economic Cooperation and Development (OECD) (2012). Education Indicators in Focus. Paris: OECD.

Organisation for Economic Cooperation and Development(OECD) (2017). Benchmarking higher education system performance: Conceptual framework and data, Enhancing Higher Education System Performance. Paris: OECD .

Programa de las Naciones Unidas para el Desarrollo (PNUD) (2018). Índices e indicadores de desarrollo humano. Actualización estadística de 2018. Nueva York: PNUD.

Pujol Riembau, Ó. (2012). El español en la India. En El español en el mundo. Anuario del Instituto Cervantes 2012. Madrid: Instituto Cervantes.

Rai, D. K. (2017). The Challenges of Spanish Language Teaching in Multilingual India: A Case Study of Delhi. Pedagogical Research, 2(2), 1-8. DOI:https://doi. org/10.20897/pr/80950

Rensburg, I., Motala, S.yArulraj David, S.(2015). Opportunitiesand challenges for research collaboration among the BRICS nations. Compare: A Journal of Comparative and International Education, 45 (5), 814-818. DOI: 10.1080/03057925.2015.1074823

Saxena, R. (2008). La enseñanza de ELE en India: Desarrollo y desafíos. Nueva Delhi: Jawaharlal Nehru University.

Sen, A. (2000). A Decade of Human Development. Journal of Human Development, $1(1), 17-23$.

Sheik, Y. A. (2017). Higher Education in India: Challenges and Opportunities. Journal of Education and Practice, 8 (1), 39-42.

The Fund for Peace (2020). Fragile States Index. Recuperado el 20 de noviembre de 2020 de https://fragilestatesindex.org/country-data/ 
United Nations Department of Economic and Social Affairs, Population Division (2019). World Population Prospects: Highlights. New York: United Nations.

United Nations, Educational, Scientific and Cultural Organization (UNESCO) (2014). BRICS Building Education for the Future. Recuperado el 20 de noviembre de 2020 de https://unesdoc.unesco.org/ark:/48223/pfoooo229692

University Grants Commission (2019). Growth of Higher Education in India. New Delhi. Recuperado el 20 de noviembre de 2020 de https://www.ugc.ac.in/stats.aspx 